\title{
PERSPECTIVAS DE LA PSICOLOGÍA DIALÉCTICA
}

\author{
THE DIALECTIC PSYCHOLOGY PERSPECTIVES \\ Ricardo Oliveros M. ${ }^{1}$ \\ Universidad Nacional Mayor de San Marcos, Perú \\ (RECIBIDO EL 06/01/2010, ACEPTADO EL 11/06/2010)
}

\begin{abstract}
RESUMEN
En el presente texto se exponen las perspectivas de la psicología dialéctica en el Perú del siglo XXI. Se reflexiona acerca de la denominación de psicología dialéctica, relacionándola con otras denominaciones que son usadas en la tendencia materialista de la psicología. Se analizan las relaciones entre psicología dialéctica y neurociencia social, delimitando el campo de las ciencias psicológicas y el campo de las neurociencias. Se desarrollan cuestiones del proyecto emancipador de la psicología dialéctica, precisando aspectos de desarrollo personal y de orientación psicológica. Se plantean nuevos aspectos del paradigma revolucionario en las ciencias del psiquismo y de la personalidad, formulando un plan de investigaciones de los temas claves de la psicología dialéctica.
\end{abstract}

Palabras clave: Psicología dialéctica; neurociencia social; proyecto emancipador; paradigma revolucionario.

\begin{abstract}
The present paper exposes the dialectic psychology perspectives in the twentieth first century Peru. We ponder about the dialectics psychology denomination, connecting them to other denominations used in the materialist psychology trend. We analyze the relations between dialectics psychology and social neuroscience, delimiting both the psychological sciences field and the neuroscience field. We develop issues from the emancipator project of dialectics psychology, precising personal development and psychological orientation aspects. We propound new aspects of the revolutionary paradigm in the psychism and personality, formulating a research plan of the key issues in dialectics psychology.
\end{abstract}

Keywords: Dialectic psichology; social neuroscience; emancipator project; revolutionary paradigm.

1 Docente asociado de la Facultad de Psicología de la UNMSM.

E-mail: oliverosr@gmail.com. 


\section{INTRODUCCIÓN}

En el presente texto se exponen las perspectivas de la psicología dialéctica en el Perú del siglo XXI, considerando algunos aspectos de la historia de la psicología dialéctica. Queda como una tarea pendiente realizar una revisión profunda de los hechos más importantes del movimiento de construcción de la psicología dialéctica en el Perú desde 1950-2010. También se reflexiona acerca de la denominación de psicología dialéctica de la persona concreta, como un tema de caracterización importante para comprender las diversas corrientes que parten de una concepción y un uso marxista de la psicología, realizando un breve análisis de las otras denominaciones usadas en la tendencia materialista de la psicología en la psicología peruana. Igualmente, se analizan las relaciones entre psicología dialéctica de la persona concreta y neurociencia social de la actividad personal, delimitando el campo de las ciencias psicológicas y el campo de las neurociencias, en tanto se presenta como una cuestión problemática para el pensamiento psicológico en el Perú. Además, se abordan aspectos relacionados con el proyecto emancipador de la psicología dialéctica de la persona concreta, en la medida en que se relacionan con la orientación del desarrollo personal y con los usos sociales del conocimiento psicológico. Finalmente, se plantean nuevos aspectos del paradigma revolucionario en las ciencias psicológicas, formulando un plan de estudio de temas claves para la psicología dialéctica de la persona concreta en el siglo XXI.

Las preguntas iniciales que nos planteamos son: ¿cuál es la importancia estratégica de la psicología dialéctica de la persona concreta en el siglo XXI? ¿Qué tipo de expectativas genera en el plano académico y qué tipo de necesidades, personales y sociales, satisface en la actividad profesional? ¿Cuáles son los temas centrales, las ideas y planteamientos claves ("núcleo duro") de la psicología dialéctica de la persona concreta?

Al respecto, nuestras reflexiones se desarrollan alrededor de tres puntos.

Primero, como crítica del academicismo en psicología. La crítica del academicismo en psicología se dirige a la falta de concreción de la psicología oficial con relación a cuestiones realmente decisivas de la vida personal. La miseria teórica de la psicología oficial comienza con la despolitización de los contenidos de la formación profesional y el vaciamiento de los contenidos significativos de la vida personal. Es realmente importante trabajar en la cuestión de los contenidos políticos de la psicología, en los usos sociales del conocimiento psicológico. Sève (1972), con relación al estudio de los problemas psicológicos de la vida real, señala la importancia de considerar la personalidad como un proceso de interiorización de las relaciones políticas presentes en la sociedad.

Segundo, como crítica de la sabiduría convencional entendida como adaptación al sistema capitalista y a la democracia burguesa. Es importante desarrollar como tema de estudio psicológico la sabiduría personal necesaria para que una persona desarrolle la conciencia de la plenitud existencial, pero es importante tarea emancipadora cuestionar la "sabiduría" como adaptación pasiva y resignada al sistema dominante. Cuestionar la supuesta madurez psicológica ("edad de la razón", de adaptarse al sistema), sobre todo cuando implica evadir una toma de posición ante cuestiones cruciales de la vida personal y social, sobre todo cuando lo fundamental se centra en una suerte de banalización de aspectos significativos de la vida personal, cuando implica pasividad existencial e indiferencia ante cuestiones vitales de la dinámica sociocultural e ideológico-política. 
Tercero, como una apuesta por el desarrollo de la creatividad personal como competencia especial de la autodinámica constructiva de la persona concreta. La autodinámica constructiva de la persona concreta implica una dialéctica de la situación vital y de formación personal en la que confluyen los procesos de socialización y los procesos de personalización. La dialéctica de la situación vital se relaciona concretamente con las condiciones personales y sociofamiliares de vida personal, considerando de manera práctica al juego, al estudio y al trabajo como actividades rectoras para orientar/organizar la gestión de la vida personal. La dialéctica de la actividad personal se relaciona con el horizonte concreto de formación personal, con la configuración práctica de ideales, convicciones, aspiraciones, etc. para el desarrollo personal en el futuro.

Respecto a los puntos señalados, en el presente texto se abordan cuatro temas relacionados: acerca de la psicología dialéctica de la persona concreta, las relaciones entre psicología dialéctica de la persona concreta y la neurociencia social de la actividad personal, el proyecto emancipador de la psicología dialéctica de la persona concreta y las perspectivas del paradigma revolucionario en las ciencia psicológicas.

\section{ACERCA DE LA PSICOLOGÍA DIALÉCTICA DE LA PERSONA CONCRETA}

La psicología dialéctica de la persona concreta se desarrolla en el marco del paradigma crítico-constructivo de la dialéctica científica, con una visión creativa del conocimientoemancipación y con el análisis concreto de la estructura y de la dinámica de las relaciones humanas en el contexto sociohistórico y cultural. La psicología dialéctica de la persona concreta utiliza la metodología de la dialéctica materialista, en las modalidades de síntesis teórica y de sistematización de la práctica, para una organización alternativa de los conocimientos psicológicos y para la formación de psicólogos como profesionales con visión de los emprendedores sociales en el siglo XXI.

Tesis 1. La psicología dialéctica de la persona concreta se desarrolla como paradigma crítico-constructivo en el marco de un proyecto emancipador del ser humano, con una visión creativa del conocimiento-emancipación y mediante un análisis concreto de la estructura y de la dinámica de las relaciones humanas en el contexto socio-histórico y cultural.

Contra el sistema de dominación, explotación y opresión del capitalismo imperialista, el paradigma crítico-constructivo implica una definida toma de posición a favor de las luchas por la emancipación humana y el desarrollo sociocultural. El paradigma críticoconstructivo utiliza creativamente la dialéctica materialista para comprender el proceso socio-histórico de la actividad vital del ser humano y para explicar la dinámica sociocultural del desarrollo personal. Con una visión creativa del conocimiento-emancipación, con perspectiva histórica y con categorías integradoras, orienta una organización alternativa de los conocimientos psicológicos con relación a intereses emancipadores. En la dialéctica de los procesos de reproducción y de transformación sociohistórica, la psicología dialéctica de la persona concreta usa a nivel psicológico el conocimiento-emancipación y genera la modalidad personal del conocimiento orientador para la gestión de la conducta personal en condiciones sociohistóricas concretas. 
Tesis 2. La psicología dialéctica de la persona concreta utiliza la metodología de la dialéctica materialista en las modalidades de síntesis teórica y de sistematización de la práctica, para una organización alternativa de los conocimientos psicológicos y para la formación de psicólogos emprendedores en el siglo XXI.

En la dimensión cultural y en la dimensión sociohistórica del desarrollo humano, la psicología dialéctica de la persona concreta promueve los intereses emancipadores y facilita una dinámica de innovación en la construcción de la vida social y cultural. Así, con relación a la dialéctica de la personalidad, la psicología dialéctica de la persona concreta promueve un modo de vida solidario para la dinámica de individuación democrática, organizando los procesos de socialización y personalización en el marco del paradigma de la solidaridad. Al mismo tiempo, con un enfoque emprendedor de la creatividad personal, la psicología dialéctica de la persona concreta lucha contra la despersonalización (efectos psicológicos de la alienación), promoviendo procesos de emancipación humana y prácticas formativas de orientación solidaria, replanteando el sentido de la vida con relación a la dinámica de cambios históricos.

En tal sentido, se puede señalar — de manera general— que la psicología dialéctica de la persona concreta es una corriente innovadora al interior de la tendencia materialista de la psicología, donde se desarrollaron varias corrientes contrarias al idealismo en psicología, sobre todo contra los usos reaccionarios de la psicología burguesa; es decir, contra una psicología que estudia sujetos sin definir claramente los contextos históricos y las condiciones sociales y culturales del desarrollo humano, sujetos descontextualizados a pesar de la importancia decisiva de los mismos para el desarrollo personal; una psicología que investiga sujetos apolíticos, sujetos aislados, sujetos sin perspectiva social y sin posición política ante los problemas de su época histórica, etc. Contrariamente, como alternativa, la tendencia materialista en psicología pone énfasis en la dimensión sociohistórica y cultural del desarrollo humano, mediante varias corrientes psicológicas que han recibido diversas denominaciones. A continuación se presentan, con una aproximación crítica inicial, algunas de las denominaciones relacionadas con el enfoque señalado:

Observaciones a la denominación de "psicología soviética". Se trataba de una caracterización inadecuada para el tipo de conocimiento científico psicológico que se había generado después de la revolución soviética. El posicionamiento de la tendencia materialista en psicología se asoció de manera incorrrecta a su "origen geopolítico", sin tomar en cuenta la cuestión cultural y la situación social real de los países a los que llegaba la información psicológica especializada. A pesar de sus aportes científicos y de su nivel teórico-conceptual no se logró generar un proceso real de apropiación social de los conocimientos producidos. Sin embargo, si se valora adecuadamente el nivel teórico-conceptual de la "psicología soviética" y se relaciona correctamente con las formas de praxis revolucionaria presentes en el contexto latinoamericano, los resultados son muy satisfactorios para el proceso de generación de conocimientos psicológicos y para la construcción de la psicología dialéctica de la persona concreta en el siglo XXI. Se tiene que realizar un trabajo de gestión conceptual y de gestión de modelos teóricos para lograrlo, en el marco de la lucha por un socialismo revolucionario y del proyecto emancipador que lo acompaña. 
Observaciones a la denominación de "psicología histórico-cultural". La caracterización como de "psicología histórico-cultural" o, también, "psicología histórico-social”, pone énfasis en lo social e histórico de los procesos psicológicos. Se trata de una caracterización asociada a la expresión de "psicología soviética" y al nombre de Vigotski. Se pierde la referencia a, por ejemplo, los aportes de la psicología de Wallon que trabaja con una concepción centrada en la explicación psicológica de la persona concreta. Se puede utilizar con relación a la psicología dialéctica de la persona concreta, especialmente con referencia al texto de Jorge Thénon y al texto de César Guardia Mayorga, Psicología del hombre concreto, en visiones actualizadas en pleno desarrollo.

Observaciones a la denominación de "psicología dialéctica". Se trata de, por decirlo así, un membrete con historia. Es el nombre de un libro de Jorge Thénon, Psicología dialéctica, un texto clásico sobre el proceso de apropiación social del conocimiento científico psicológico más avanzado. Un ejemplo de generación de conocimiento científico psicológico que responde a las necesidades y tareas de la realidad psicológica latinoamericana, un texto clásico desde la década de los 70. La denominación "psicología dialéctica” se asoció en el Perú a la creación de una asignatura con ese nombre para generar una alternativa en el plano académico a lo que representaba el conductismo y el psicoanálisis, en un contexto político-social de lucha política por el socialismo se trata de una toma de posición acerca de los aportes de la psicología al proyecto emancipador del pueblo peruano. Se discutían no solamente sobre las tareas de los intelectuales revolucionarios, sino acerca de la función de los profesionales de la psicología con relación al proceso de emancipación social.

Observaciones a la denominación de "psicología materialista". La expresión "psicología materialista" o mejor aún "tendencia materialista en psicología” se utilizaba en varios textos y se hacía referencia en los debates académicos. Uno de los autores que se expresaban así era Leontiev en su texto Actividad, conciencia y personalidad. Si se trataba de, como solía decirse en los años 70, sentar posición era una expresión pertinente. Se dejaba establecida la existencia de dos tendencias (la materialista y la idealista) y eso implicaba un posicionamiento polémico en el campo académico de la psicología y se expresaba en estrategias para las prácticas formativas de los psicólogos. Las tareas de enseñanza y aprendizaje de la psicología implicaban una toma de posición filosófica y existencial. No todos asumían la tarea señalada con la claridad y la profundidad que una toma de posición semejante implicaba en la formación personal y en el desarrollo profesional. Se trata de profundizar este enfoque en el estudio de la psicología en el Perú, en la medida en que el problema sigue vigente y para avanzar se tiene que formular una posición adecuada tanto para el proceso de apropiación social del conocimiento científico psicológico, como para el proceso de producción de conocimiento científico psicológico desde nuestra realidad psicológica, en el marco del proyecto emancipador del socialismo peruano en construcción.

Observaciones a la denominación de “psicología marxista”. La caracterización de "psicología marxista" es sumamente importante analizarla en perspectiva y con claridad de propósitos científico-profesionales, además de los político-sociales y culturales. Habría que precisar, por ejemplo, la diferencia entre "posición marxista" y "posición socialista", en la medida en que se relacionan pero no son lo mismo y existen claras diferencias en más de un aspecto. Se puede analizar la concepción marxista de hombre y también 
la concepción socialista de hombre. Se puede hablar de un marxismo humanista o del humanismo científico del marxismo. Se puede hablar del socialismo revolucionario, del socialismo democrático y del humanismo socialista. En este contexto, ciertas versiones del psicoanálisis se declaraban de orientación marxista, incluso a ciertos conductistas se les antojó denominarse revolucionarios. A decir verdad, una cuestión abierta al debate en el campo de las ciencias psicológicas.

En resumen, las denominaciones señaladas nos presentan un vasto campo de cuestiones que son claves como marco del desarrollo de la psicología dialéctica de la persona concreta. Las confusiones y expectativas equivocadas, también los miedos innecesarios y los prejuicios existentes se podrían evitar si se desarrollaran debates académicos sobre los temas en cuestión. La creación de un discurso sobre las cuestiones que superan y, al mismo tiempo, sirven de marco orientador para la tarea de construcción de la psicología dialéctica de la persona concreta en el siglo XXI requiere de claras tomas de posición y de debates académicos. Los posicionamientos necesarios que se tienen que dar son, por ejemplo, respecto a la concepción de hombre, al proyecto emancipador del socialismo peruano, a las concepciones sobre el florecimiento humano y la lucha contra las formas de enajenación, a los enfoques acerca del trabajo humano como cuestión central del desarrollo personal en las sociedades de clases, y a la lucha contra la explotación, etc. Sin posiciones claras y argumentadas no hay construcción de un discurso académico-profesional de la psicología dialéctica de la persona concreta. Sin un programa de trabajo colectivo centrado en la construcción de la psicología dialéctica de la persona concreta en el siglo XXI no hay estrategias de posicionamiento científico-profesional, ni de posicionamiento sociocultural con una clara perspectiva histórica y revolucionaria.

\section{Psicología dialéctica de la persona concreta y neurociencia social de la actividad personal de orientación dialéctica}

En el contexto peruano, el proceso de generación de conocimiento psicológico está asociado a la delimitación del campo de estudio de las disciplinas científicas, delimitando el campo de las ciencias psicológicas y el campo de las neurociencias. Específicamente, las relaciones entre la psicología dialéctica de la persona concreta y la neurociencia social de la actividad personal, con orientación dialéctica.

Para comenzar, he planteado (2005) que el modelo teórico acerca de la estructura de la actividad consciente de la persona, desarrollado por Pedro Ortiz Cabanillas, es el núcleo de estructuración de la psicología dialéctica de la persona concreta. Se trata de un planteamiento que implica el desarrollo de una serie de cuestiones teóricas. Se trata de explicar la distribución de las disciplinas en el campo de las neurociencias y en el campo de las ciencias psicológicas, considerando el modelo teórico en cuestión como una matriz teórica en expansión, que es lo que he planteado en un artículo (Oliveros, 2009). Se trata de explicar que el modelo teórico se relaciona con cuestiones acerca de la teoría del hombre, como una versión especial de la comprensión de lo humano y de la vida, y también se relaciona con cuestiones sobre el sistema de la actividad humana. Se trata de comprender los aportes a las ciencias directamente relacionadas con el ser humano, desde la generación de conocimiento científico, para la estructuración y el desarrollo de 
diversas disciplinas científicas en el campo de las neurociencias y también en el campo de las ciencias psicológicas.

Por lo señalado, se puede decir que el "modelo teórico" de Ortiz, como "matriz teórica en expansión" está generando interpretaciones diversas y promoviendo diversos desarrollos. Al respecto nos preguntamos: ¿cómo se generan y estructuran las interpretaciones teóricas en los diversos campos del saber científico? ¿Cuál es la estructura del conocimiento científico de una disciplina científica? ¿Qué es lo que dinamiza - genera cambios y desarrollo- la generación y producción de conocimiento científico en un determinado campo del saber? Se trata de preguntas importantes porque la definición de una tendencia en el campo de las ciencias psicológicas, y también la presencia de corrientes específicas dentro de la misma tendencia, es una tarea importante de reflexión acerca del conocimiento psicológico, es parte del desarrollo de las tareas teóricas en el proceso de construcción de la psicología en el siglo XXI.

¿Qué lugar ocupan —como disciplinas científicas - la neurofisiología y neuropsicología en las neurociencias y en las ciencias psicológicas? ¿Qué es y cómo se configura una disciplina científica en el campo de las neurociencias? ¿Cuáles son las principales disciplinas científicas en el campo de las neurociencias? ¿Cuáles son las relaciones entre disciplinas científicas que solamente cambian el prefijo?, por ejemplo: ¿neurofisiología y psicofisiología?, ¿neurolingüística y psicolingüística?, ¿neurocultura y psicocultura?, ¿neurohistoria y psicohistoria?, ¿neuropolítica y psicopolítica?, ¿neuropatología y psicopatología?, ¿neuropsicología de la personalidad y psicología de la personalidad?, ¿neurofarmacología y psicofarmacología?, ¿neurobiología y psicobiología?, etc. ¿Cuáles son las disciplinas científicas en el campo de las neurociencias y en el campo de las ciencias psicológicas?

¿Qué es lo que determina que una disciplina científica pertenezca al campo de las neurociencias o al campo de las ciencias psicológicas, en qué consiste la diferencia? Por ejemplo, ¿cuáles son las diferencias entre neurofisiología y psicofisiología? ¿Cuáles son las diferencias entre neurolingüística y psicolingüística? ¿Cuáles son las diferencias entre neuropatología y psicopatología? ¿Se puede considerar a la psicobiología como una disciplina científica que relaciona a las neurociencias y a las ciencias psicológicas? ¿Cómo queda la denominada sociobiología del comportamiento animal y humano?

¿Qué es lo que aporta la teoría científica del hombre a la estructuración teórica de las disciplinas en el campo de las neurociencias? ¿Qué es lo que aporta la teoría científica del hombre a la estructuración teórica de las ciencias psicológicas? ¿Cuáles son los principales aportes de las neurociencias y de las ciencias psicológicas a la comprensión y mejor explicación del sistema de la actividad humana y del sistema de la personalidad?

\section{Acerca del proyecto emancipador de la psicología dialéctica de la persona concreta}

Las cuestiones relacionadas con el proyecto emancipador de la psicología dialéctica de la persona concreta tienen que plantearse con perspectiva histórica y con visión de futuro, precisando siempre su importancia estratégica para definir el horizonte de construcción teórica y la dimensión de las tareas teóricas a desarrollar. 
Para comenzar, quiero aportar un testimonio personal respecto al proyecto emancipador de la psicología dialéctica de la persona concreta. En mi historia de vida personal tengo presente con toda claridad que asumí una tarea estratégica: difundir los aportes de la tendencia materialista de la psicología para construir una psicología revolucionaria en el Perú. Al respecto, recuerdo también claramente mi encuentro con César Lévano, en la librería Cosmos. En una comunicación breve y directa me indicó la importancia del ensayo de Gonzáles Moreyra: Lenin, Mariátegui y la psicología en el Perú. Del “joven psicólogo peruano" al decir de Lévano en 1977. A mi parecer se trata de un proyecto abandonado, una toma de posición que no tuvo desarrollo, el esbozo de un plan de trabajo que se dejó de lado. Se puede decir que el proyecto emancipador de una (posible) psicología dialéctica fue abandonado. ${ }^{2}$

En ese tiempo, en las condiciones sociohistóricas concretas de la década del 70, hubiese sido sumamente importante que se pudieran plantear como elementos básicos la relación Mariátegui y la psicología dialéctica. El contenido del texto ameritaba futuro desarrollo, tanto en el campo de las ciencias psicológicas, como en el campo de la construcción del socialismo revolucionario a través del proyecto emancipador de la psicología dialéctica. Se trata de algo en lo que se tiene que volver a pensar y sobre todo desarrollar a un nuevo nivel, en las condiciones sociohistóricas concretas del siglo XXI como "tiempo de revolución" por los aniversarios de la independencia de los poderes coloniales hace 200 años. La fuente principal de ideas y planteamientos relacionados con el proyecto emancipador de la psicología dialéctica viene del pensamiento de Mariátegui, todos los debates de la izquierda peruana giraban en torno al pensamiento de Mariátegui y uno de los psicólogos más polémicos que ha tenido la Universidad Nacional Mayor de San Marcos no fue la excepción a la regla. Es decir, intervino en el tema central de los debates, en el tema marco de las reflexiones sobre la importancia social de la psicología y acerca de los usos liberadores que debería tener la psicología en el proceso revolucionario. Con todas las diferencias del caso, honor al mérito y homenaje al aporte que dejó para la historia del pensamiento psicológico peruano. La producción de subjetividad revolucionaria es una tarea que se tiene que asumir como tarea clave del proyecto emancipador, especialmente de la psicología dialéctica de la persona concreta. Ahora bien, ¿qué otras tareas claves tiene el proyecto emancipador? Lucha contra las formas de alienación social e individual, combate al sentido común neoliberal, crítica de las ideologías prácticas del neoliberalismo, etc.

En la misma perspectiva, la gestión estratégica de la herencia social es una tarea clave del proyecto emancipador de la psicología dialéctica de la persona concreta. Por ejemplo, ¿qué podemos aprender de la Psicología del hombre concreto de César Guardia Mayorga? Quizá lo mejor de la "herencia social” para la psicología dialéctica no está precisamente en su libro de psicología. Sabía filosofía, sabía quechua, sabía la importancia de la cultura, etc. con relación al "hombre concreto".

La gestión de la herencia social es una cuestión clave de la historia de la psicología. Algunas veces, simplemente un orden diferente de las cuestiones puede cambiar la historia de un objeto teórico o la orientación de una práctica profesional. Por ejemplo, orientaciones

2 La publicación del ensayo psicológico de Gonzalez Moreyra (1970): "Lenin, Mariátegui y la psicología", con notas y comentarios está en preparación. Se trata de un importante documento histórico para profundizar en la historia de la psicología dialéctica en el Perú. 
psicológicas que se desarrollan en el marco de un proyecto emancipador y orientaciones psicológicas que privilegian prácticas profesionales individualizadas con un enfoque psicologista (es decir, que consideran débilmente y en formas sumamente restringidas el entorno del individuo).

En tal sentido, el proyecto emancipador se configura como un marco de orientación de las prácticas formativas y de las prácticas sociales. Se relaciona con el funcionamiento cotidiano y la orientación estratégica de las prácticas sociales y formativas con un enfoque centrado en la emancipación humana. Los temas claves de la psicología dialéctica se definen con relación al proyecto emancipador. Se trata de una cuestión decisiva para la gestión del conocimiento orientador que emerge de los aportes de las disciplinas científicas. La memoria histórica se reconstruye alrededor de los supuestos fundamentales del proyecto emancipador y las formas concretas de expresión en el campo de acción de las ciencias psicológicas.

Ahora bien, ¿cuál es el problema central en cuestión con el proyecto emancipador de la psicología dialéctica de la persona concreta? Simplemente que la sabiduría convencional de la psicología oficial no soporta pensar temas histórico-sociales y culturales de importancia para la construcción de alternativas en el campo de las ciencias psicológicas. Por ejemplo, José María Arguedas: socialismo mágico y emancipación humana, desde la visión de la psicología dialéctica de la persona concreta. Al respecto, nos preguntamos y lanzamos el desafío: ¿cómo superar los efectos psicológicos del colonialismo interno y de la dominación del capitalismo imperialista? ¿Cómo explicar las formas de individualidad y el desarrollo personal en las condiciones sociales de subdesarrollo de la sociedad peruana? ¿Cómo superar las formas de alienación que impiden la emancipación humana en las condiciones sociales de subdesarrollo de la sociedad peruana? ¿Cuál es el significado y el sentido del pensamiento/conocimiento situado que genera Arguedas en el plano antropológico y literario? ¿Cuál es el significado y el sentido del pensamiento/conocimiento situado que tiene que seguir desarrollando la psicología dialéctica de la persona concreta, con mayores (mediante la interdisciplinariedad) y mejores (a través del estudio y la investigación interdisciplinaria) niveles de concreción en las prácticas formativas de las personas y al servicio de las prácticas sociales de los movimientos sociales?

\section{La psicología dialéctica de la persona concreta como paradigma revolucionario}

El saber colonial y el conocimiento para la dominación ha sido casi naturalizado por la sabiduría convencional de la psicología oficial. Nos dan una perspectiva de producción del conocimiento, al margen de los procesos sociales reales. Obviamente, desde la psicología dialéctica de la persona concreta, la cuestión metodológica de la "asimilación crítica" y también las tareas de "aplicación creadora" del conocimiento científico psicológico adoptan nuevos significados y sentidos en el marco de un proyecto emancipador. ¿Qué es lo que asimila críticamente y cómo se relaciona con la tarea revolucionaria de formación personal y emancipación humana? ¿Qué es lo que se aplica creadoramente y al servicio de qué objetivos estratégicos? Por lo señalado consideramos que, definitivamente, la noción/ programa de trabajo Proyecto Emancipador genera una agenda abierta, con un horizonte definido para orientar/organizar el proceso de producción del conocimiento y también con 
un horizonte de formación no solamente de militantes intelectuales sino de nuevos tipos de intervención profesional, con modelos de conducta personal y de desarrollo personal.

Lo señalado nos plantea la importancia de asimilar la dialéctica científica para la construcción de la psicología dialéctica de la persona concreta. Una dialéctica científica operando en un doble movimiento relacionado, como pensamiento dialéctico situado y como razonamiento concreto que considera las tareas sociohistóricas y culturales relacionadas con el horizonte de formación personal y de desarrollo humano de un pueblo. En tal sentido, el proyecto emancipador de la psicología dialéctica de la persona concreta nos plantea nuevas tareas de innovación y desarrollo respecto a las formas de generación de conocimiento psicológico y a la producción de conocimiento científico en el campo de las ciencias psicológicas. Se trata de formular, con una perspectiva nueva y con un enfoque revolucionario un plan de investigaciones de los temas claves de la psicología dialéctica de la persona concreta.

¿Cómo reconstruir una psicología al servicio de las luchas populares, cómo construir una psicología dialéctica al servicio de la emancipación psicológica del pueblo? Nadie dice nada, por ejemplo cuando se habla de psicología y democracia, desde la perspectiva de la sabiduría convencional burguesa de la psicología oficial. Muchos se inquietan y protestan cuando se habla, por ejemplo, de "psicología dialéctica de la persona concreta: proyecto emancipador y socialismo revolucionario". Es importante precisar los aportes de la politización de la psicología al desarrollo del conocimiento psicológico. Se trata de una tarea pendiente con relación a la determinación de los fundamentos concretos y a la dinámica práctica de los dos componentes adicionales que propone Martiza Montero para una comprensión integral del paradigma científico.

¿Cómo explicar concretamente una situación compleja, especialmente con relación al movimiento de construcción de la psicología dialéctica de la persona concreta? Para comenzar, es importante realizar la mejor y más completa caracterización de la situación, concentrándose en la dinámica práctica de la situación problemática y analizando la conducta de los protagonistas principales, considerando el contexto y las expresiones principales de la lógica del poder. Se tiene que captar la estructura de los procesos y la dinámica práctica de las contradicciones que generan el movimiento y sus manifestaciones en tendencias, corrientes, etc. Una nueva situación (como entramado complejo de tendencias y procesos) abre perspectivas nuevas y genera nuevos enfoques para hechos pasados. La caracterización de una situación es sumamente importante. Se tiene que captar la dialéctica de la situación para darle un tratamiento adecuado a las tendencias existentes y para comprender la manifestación de los procesos en pleno desarrollo. La gestión de la realidad implica una gestión del conocimiento de la situación y una gestión de la actuación de la personalidad. Los protagonistas involucrados en una situación toman decisiones y actúan de acuerdo a todos los aspectos señalados. El desenlace de una situación problemática depende de la gestión de la realidad, de la gestión del conocimiento y de la gestión de la actuación personal.

¿Cómo se relaciona la dialéctica de la situación con los procesos relacionados con la gestión de la realidad, por ejemplo, en el proceso de definición del campo de las neurociencias y del campo de las ciencias psicológicas? Dos planos relacionados: conocimiento de la 
realidad y transformación de la realidad; un solo proceso en desarrollo permanente: gestión de la realidad mediante el compromiso y el involucramiento de los actores principales, a través de la comprensión de la lógica de actuación de la personalidad de los protagonistas, por ejemplo, destacando el desarrollo de la teoría sociohistórica del florecimiento humano como alternativa a las teorías de la alienación y al servicio del proyecto emancipador de la psicología dialéctica de la persona concreta.

La gestión del conocimiento de la realidad es clave para facilitar el desenlace de una situación problemática. Al mismo tiempo es importante realizar adecuadamente una caracterización tendencial de la actuación personal de los principales protagonistas de una situación problemática. No solamente para evitar que las contradicciones del proceso en cuestión se agudicen, sino para lograr la solución de los problemas y de los conflictos generados. Al respecto, ¿por qué no generamos modelos y estrategias de formación científica y de actuación profesional con un horizonte emancipatorio para el siglo XXI?

Se trata de construir modelos y desarrollar estrategias para la formación científica en psicología y tipos de emprendizaje social para la actuación profesional en psicología con el enfoque de los emprendedores sociales en el campo de las ciencias psicológicas. Se trata de construir el discurso del proyecto emancipador de la psicología dialéctica de la persona concreta, un discurso que se relaciona con la lucha por el socialismo. Se trata de formular y desarrollar el programa de trabajo del proyecto emancipador, considerando las perspectivas que define y precisando las tareas que señala para el desarrollo de la psicología dialéctica de la persona concreta en el siglo XXI.

¿Qué perspectivas define el proyecto emancipador para la construcción de la psicología dialéctica de la persona concreta? ¿Qué tareas señala el proyecto emancipador para el desarrollo de la psicología dialéctica de la persona concreta? Al respecto, un texto clave es Filosofía de la praxis de Adolfo Sanchez Vasques. De manera especial, en el Perú estamos ante la comprensión integral del pensamiento de Mariátegui. Se trata de destacar las cuestiones psicoculturales, la dimensión cultural del desarrollo humano en la construcción de la psicología dialéctica. La psicología de la cultura es una cuestión clave a considerar, pensando en un modelo emprendedor de formación profesional centrado en la creatividad personal y en el emprendizaje.

\section{CONCLUSIONES}

- La formación de mentalidades y/o configuración de subjetividades (sociales y personales) es un tema clave de la reflexión teórica de la psicología dialéctica de la persona concreta y de su posicionamiento académico.

- La superación de la alienación social, como problema sociohistórico y psicocultural, es una tarea clave de la práctica formativa de la psicología dialéctica de la persona concreta.

- La psicología dialéctica de la persona concreta, como paradigma crítico-constructivo en las ciencias psicológicas, es el resultado del debate académico y de la construcción teórica de las ciencias psicológicas de orientación científica. 
- La psicología dialéctica de la persona concreta centra el debate académico en propuestas relacionadas con la realización del programa de trabajo de un proyecto emancipador.

- La característica distintiva de la psicología dialéctica de la persona concreta es su orientación a la realización de su proyecto emancipador, con una perspectiva sociohistórica del saber y un enfoque dialéctico del conocimiento científico.

- La orientación ético-política de la psicología dialéctica de la persona concreta es el eje para el desarrollo de estrategias de posicionamiento académico y profesional. Es importante trabajar intensamente en este asunto.

- La psicología dialéctica de la persona concreta, genera actividad reflexiva y memoria histórica, con relación al proceso de reorientación de prácticas formativas y el proyecto emancipador para la realización del florecimiento humano de todos.

\section{REFERENCIAS BIBLIOGRÁFICAS}

1. Oliveros Mejía, Ricardo (2005). El proyecto emancipador de la psicología dialéctica. En Revista de investigación en psicología, Instituto de investigaciones psicológicas, UNMSM, 8, 2, 151-160.

2. Oliveros Mejía, Ricardo (2008). Neurociencia y psicología: formación de la personalidad en el siglo XXI. En Revista peruana de psicología, Colegio de psicólgos del Perú, 2, 2-2008, 235-251.

3. Ortiz Cabanillas, Pedro (1994). El sistema de la personalidad. Lima: Orión.

4. Ortiz Cabanillas, Pedro (1997). La formación de la personalidad. Lima: Damaso Editores.

5. Ortiz Cabanillas, Pedro (2002). Lenguaje y habla personal. Lima: Fondo Editorial UNMSM.

6. Ortiz Cabanillas, Pedro (2004). El nivel consciente de la actividad personal. Lima: Fondo Editorial UNMSM.

7. Ortiz Cabanillas, Pedro (2008). Educación y formación de la personalidad. Lima: Fondo Editorial de la Universidad de Ciencias y Humanidades.

8. Sanchez Vasques, Adolfo (2003). Filosofía de la praxis. México: Editorial FCE.

9. Sève, Lucién (1972). Marxismo y teoría de la personalidad. Argentina: Editorial Amorrortu.

10. Thénon, Jorge (1974). Psicología dialéctica. Argentina: Editorial Paidós. 